\title{
Estimating confidence intervals for eigenvalues in exploratory factor analysis
}

\author{
Ross LARSEN ANd Russell T. WARne \\ Texas A\&M University, College Station, Texas
}

\begin{abstract}
Exploratory factor analysis (EFA) has become a common procedure in educational and psychological research. In the course of performing an EFA, researchers often base the decision of how many factors to retain on the eigenvalues for the factors. However, many researchers do not realize that eigenvalues, like all sample statistics, are subject to sampling error, which means that confidence intervals (CIs) can be estimated for each eigenvalue. In the present article, we demonstrate two methods of estimating CIs for eigenvalues: one based on the mathematical properties of the central limit theorem, and the other based on bootstrapping. References to appropriate SAS and SPSS syntax are included. Supplemental materials for this article may be downloaded from http://brm.psychonomic-journals.org/content/supplemental.
\end{abstract}

Exploratory factor analysis (EFA) is a statistical procedure that reduces the number of observed variables into a smaller number of latent variables - called factors - that account for most of the variance of the original observed variables. Originated by Spearman (1904), EFA allows a researcher to determine the number and nature of groupings that the observed variables fall into. Over the course of the past century, EFA has proven to be useful in test development, evaluating validity, developing theory, and computing factor scores for use in subsequent analyses (e.g., regression, descriptive discriminate analysis).

The use of EFA has increased in recent years in the published empirical research. In the midtwentieth century, prior to widespread availability of hardware and software, only $4 \%$ of empirical studies used EFA (Edgington, 1964, p. $202 ; 1974$, p. 25). By the late 1980 s, that proportion had almost doubled to $7.6 \%$ (Elmore \& Woehlke, 1988, p. 19). More recent studies have shown that between $12.7 \%$ and $27 \%$ of empirical research articles in psychology journals on individual differences - that is, journals concerned with correlational psychology (Cronbach, 1957) —used EFA (Fabrigar, Wegener, MacCallum, \& Strahan, 1999, p. 292; Russell, 2002, p. 1629).

In the course of performing an EFA, a researcher must make many decisions. These decisions include choosing a factoring method and a rotation procedure, and interpreting the results (see Gorsuch, 1983, and Thompson, 2004, for a full treatment of the steps of an EFA). However, the most important decision that a researcher makes when conducting an EFA is choosing the number of factors to retain (Costello \& Osborne, 2005; Zwick \& Velicer, $1982,1986)$. The decision is so important that "assorted decisions on the other issues [in an EFA] generally produce similar results when the optimal number of factors is specified" (O'Connor, 2000, p. 396).
Most of the methods that drive the decision of how many factors to retain are largely determined by the eigenvalues of each factor. For example, the Guttman (1954) rule requires researchers to retain all factors for which the eigenvalue is greater than 1.0. The scree test (Cattell, 1966) also uses eigenvalues, which are plotted in a graph. The resulting graph is sometimes shaped like an elbow, and all eigenvalues before the bend are usually retained. These two methods of deciding how many factors to retain in an EFA are by far the most commonly used methods in the published literature (Fabrigar et al., 1999; Ford, MacCallum, \& Tait, 1986; Hayton, Allen, \& Scarpello, 2004; Henson \& Roberts, 2006). A more complex - and more accurate (Zwick \& Velicer, 1986) - method of deciding how many factors to retain, parallel analysis (Horn, 1965), is also dependent on observed eigenvalues.

Researchers who use these methods of deciding how many factors to retain may not sufficiently consider that eigenvalues - like all statistics based on sampling - are susceptible to sample error. This means that eigenvalues are mere point estimates and that confidence intervals (CIs) can be constructed around them (Thompson, 2004; Zientek \& Thompson, 2007). The purpose of the present article is threefold. First, we will explain why it is important to report the CIs for eigenvalues. Second, we intend to present two methods of estimating CIs for eigenvalues and provide SPSS and SAS code to calculate CIs. Third, we will discuss potential future applications of eigenvalue CIs in determining the number of factors to retain.

Before demonstrating the two methods of estimating eigenvalue CIs, we wish to explain our terminology. In the present article, we use the term exploratory factor analysis to refer to both principal components and common factor analysis. We are aware of the long-standing controversy in the field about the two methods (Gorsuch,

R. Larsen, ross.larsen@dsmail.tamu.edu 
2003) and that some readers will disagree strongly with our broad use of this term. We are neutral in the argument over the preference for a particular method and whether one or the other constitutes a "real" factor analysis. To accommodate both sides of the debate, we have ensured that the SAS and SPSS syntax that we refer to in the present article can be adapted to either extraction method. However, the example analysis that we perform is a principal components analysis.

\section{REASONS FOR ESTIMATING EIGENVALUE CIs}

CIs are a specified range of plausible values for a population parameter of interest. This is opposed to simply reporting the point estimate of a statistic, such as a mean or observed eigenvalue. CIs convey important information about the precision of population parameter estimates that is not present in a point estimate (Wilkinson \& the Task Force on Statistical Inference, 1999). A very common type of CI is the $95 \% \mathrm{CI}$, although other levels of confidence $(0<x \%<100)$ are possible.

Because CIs for eigenvalues are so rarely reported, some readers may question why the CIs should warrant a researcher's attention at all. The first reason why we believe that CIs should be reported for eigenvalues is because of the new reporting standards that have been produced by the American Psychological Association (APA). Although in previous years CI reporting was recognized as good practice (see, e.g., Cumming \& Finch, 2005; Wilkinson \& Task Force on Statistical Inference, 1999; Zientek, Capraro, \& Capraro, 2008), the latest version of the APA publication manual mandates that CIs always be reported (APA, 2010). We believe that this mandate extends to eigenvalues and that the present article can be a helpful guide to researchers in the future. Moreover, reporting the eigenvalue CIs provides more information to the reader and increases transparency in EFA research.

Second, CIs for eigenvalues can serve as an enhancement to traditional methods of deciding the number of factors to retain. The most common methods of choosing the number of factors to retain are the Guttman (1954) rule and the scree test (Cattell, 1966). As was mentioned previously, the Guttman rule requires researchers to retain all eigenvalues with observed eigenvalues greater than 1.0 , which is based on the reasonable requirement that a retained factor should explain more variance than an observed variable does. In a scree test, one looks for a sharp "break" in the slope of eigenvalues, which represents the point at which meaningless factors start. The researcher therefore retains all of the factors that come before the horizontal line of the final meaningless factors.

However, neither of these methods takes into consideration the fact that eigenvalues are sample statistics that can have CIs. For example, it has long been observed that the Guttman rule's strict retention of all factors with an eigenvalue greater than 1.0 is arbitrary (Fabrigar et al., 1999; Ford et al., 1986; Loehlin, 2004; Thompson, 2004). Researchers who calculate CIs for their eigenvalues may encounter a factor whose CI includes values above and below 1.0, which leads to the conclusion that a strict application of the Guttman rule is not defensible. A more thoughtful use of the Guttman rule may be to retain factors for which the entire eigenvalue $\mathrm{CI}$ is greater than 1.0 - a version of the Guttman rule that cannot be applied without calculating eigenvalue CIs.

Likewise, instead of creating a scree plot based on observed eigenvalues, researchers could produce a series of box plots constructed from the eigenvalues' CIs, similar to the ones created by Zientek and Thompson (2007). However, box plots for the eigenvalues may be less likely to form a clean break in the pattern and may make the scree test difficult to apply. Therefore, the use of eigenvalue CIs helps to demonstrate one of the major shortcomings of the scree test, and the presence of CIs may compel a researcher to use caution with scree plots or to use a different method of choosing the number of factors to retain.

Eigenvalue CIs also have potential applications in parallel analysis (Horn, 1965). In Horn's original version of parallel analysis, one compares observed eigenvalues with those obtained from randomly generated data and retains all of the factors for which the observed eigenvalue is greater than the corresponding randomly generated eigenvalue. In other incarnations of parallel analysis (e.g., Glorfeld, 1995; O'Connor, 2000), the CIs are calculated through bootstrapping for the random data's eigenvalues. The observed eigenvalues are compared with the entire CI for the random data, and all factors with an eigenvalue greater than the entire CI are retained. We propose a variation of parallel analysis that could use CIs for observed eigenvalues and retain factors with a random data eigenvalue lower than the entire corresponding observed eigenvalue $\mathrm{CI}$ range.

Some researchers may also compare CIs for both the random data and observed data and retain only those factors with completely nonoverlapping CIs. However, it should be noted that this latter method is very conservative, with an approximate $p$ value of .006 when both CIs are $95 \%$ CIs (Cumming \& Finch, 2005, p. 176), whereas the previous two applications theoretically correspond to $p=.05$.

However a researcher chooses to use eigenvalue CIs in his or her research, we believe that they are a vital part of the EFA process and should no longer be ignored. The methods of estimating the CIs and the SAS and SPSS syntax that follow are designed to make this added practice in an EFA as accessible as possible for researchers.

\section{TWO METHODS FOR CONSTRUCTING EIGENVALUE CIS}

Two methods can be used to estimate CIs for eigenvalues. The first method is an equation based on the properties of the central limit theorem. The second method is to use bootstrapping to construct CIs. Because bootstrapping methods are computationally intensive, we have included references to useful SAS and SPSS syntax.

We have also included sample results of each method in Table 1. Following the lead of Zientek and Thompson (2007), we are using nine variables from the archival data 
Table 1

95\% Confidence Intervals (CIs) for Eigenvalues of Selected Data From Holzinger and Swineford (1939)

\begin{tabular}{ccccc}
\hline $\begin{array}{c}\text { Factor } \\
\text { Number }\end{array}$ & $\begin{array}{c}\text { Observed } \\
\text { Eigenvalue }\end{array}$ & Equation 9 CI & $\begin{array}{c}\text { Bootstrap Syntax } \\
\text { (SAS) CI }\end{array}$ & $\begin{array}{c}\text { Bootstrap Syntax } \\
\text { (SPSS) CI }\end{array}$ \\
\hline 1 & 2.945 & {$[2.474,3.417]$} & {$[2.623,3.301]$} & {$[2.634,3.344]$} \\
2 & 1.778 & {$[1.494,2.063]$} & {$[1.543,2.209]$} & {$[1.536,2.032]$} \\
3 & 1.396 & {$[1.173,1.620]$} & {$[1.186,1.590]$} & {$[1.210,1.581]$} \\
4 & 0.717 & {$[0.602,0.831]$} & {$[0.648,0.853]$} & {$[0.647,0.850]$} \\
5 & 0.629 & {$[0.529,0.730]$} & {$[0.551,0.706]$} & {$[0.546,0.707]$} \\
6 & 0.535 & {$[0.450,0.621]$} & {$[0.465,0.602]$} & {$[0.466,0.602]$} \\
7 & 0.478 & {$[0.402,0.555]$} & {$[0.388,0.519]$} & {$[0.388,0.522]$} \\
8 & 0.289 & {$[0.242,0.335]$} & {$[0.241,0.337]$} & {$[0.240,0.343]$} \\
9 & 0.251 & {$[0.211,0.291]$} & {$[0.199,0.274]$} & {$[0.195,0.274]$} \\
\hline
\end{tabular}

Note-Sample size for all CIs is 301 . Original eigenvalues were obtained through an exploratory factor analysis using principal components analysis to extract the factors, which was the procedure used by Zientek and Thompson (2007). Both bootstrapping methods used 1,000 resamplings. The archival data used for this analysis are available in the supplement for this article.

of Holzinger and Swineford (1939). This data set consists of academic testing data of 301 American seventh- and eighth-grade students and is available in the supplement for this article. The variables in the present article use the scores from the following variables: T6 (paragraph completion), T7 (sentence completion), T9 (word meaning), T10 (speeded addition), T12 (speeded counting of dots in shapes), T13 (speeded discrimination of straight and curved caps), T14 (memory of target words), T15 (memory of target numbers), and T17 (memory of objectnumber association targets). We decided to follow Zientek and Thompson so closely because their SPSS syntax is ideal for calculating CIs for eigenvalues. By using the same data for the other methods, we hope that the reader will be able to adapt Thompson and Zientek's syntax to their EFAs.

\section{Mathematical Method}

To understand how to mathematically construct CIs for eigenvalues, it is necessary to review some basic multivariate statistics (Mardia, Kent, \& Bibby, 1979). Spectral decomposition is defined thus: Any symmetric matrix A can be decomposed as $\Gamma \Delta \Gamma^{\prime}$, where $\Delta$ is a diagonal matrix of the eigenvalues of $A$, and $\Gamma$ is an orthogonal matrix whose columns are standardized eigenvectors. Take $\mathrm{A}=\Sigma$, where $\Sigma$ is the variance covariance matrix of any data set, then we have

$$
\Gamma^{\prime} \Sigma \Gamma=\Delta,
$$

where $\Delta$ is a diagonal matrix of the (ordered) eigenvalues of $\Sigma$, and $\Gamma$ is a $p \times p$ orthogonal matrix. Also note that

$$
\chi_{m}^{2}=\sum_{i=1}^{m} \chi_{1 i}^{2}
$$

where $\chi_{1 i}^{2}$ are independent, thus

$$
\chi_{m}^{2} \cong \operatorname{Normal}(m, 2 m)
$$

by the central limit theorem (Rencher \& Schimek, 1995). The multivariate case is similar using the Wishart $\left(W_{p}\right)$ distribution, where $p$ is the number of parameters. If $\mathrm{M}_{1} \sim$ $W_{p}\left(\Sigma, n_{1}\right), \mathrm{M}_{2} \sim W_{p}\left(\Sigma, n_{2}\right)$ are independent,

$$
\mathrm{M}_{1}+\mathrm{M}_{2} \sim W_{p}\left(\Sigma, n_{1}+n_{2}\right) .
$$

Another way to think of it is

$$
W_{p}(\Sigma, m)=\sum_{i=1}^{m} W_{p}(\Sigma, 1) .
$$

We know that $n \mathbf{S} \sim W_{p}(\Sigma, n-1)$ (Rencher \& Schimek, 1995), which is in accordance with Rippe's (1953) work on the distribution of eigenvalues. From Equation 1, we can define

$$
n \mathrm{D}=n \mathbf{G}^{\prime} \mathbf{S G} \approx n \boldsymbol{\Gamma}^{\prime} \mathbf{S} \boldsymbol{\Gamma} \sim W_{p}(\Delta, n-1) .
$$

Let $\mathbf{d}$ denote the $p$ vector of sample eigenvalues estimated from the data and $\lambda$ be the population eigenvalues, so that

$$
n^{1 / 2}(d-\lambda) \stackrel{D}{\rightarrow} N_{p}\left(0,2 \Delta^{2}\right) .
$$

So, for each individual eigenvalue, have $l_{i}$ be the sample eigenvalue. We can thus simplify:

$$
n^{1 / 2}\left(l_{i}-\lambda_{i}\right) \stackrel{D}{\rightarrow} N\left(0,2 \lambda_{i}^{2}\right) .
$$

Then, from Slutsky's theorem and its extensions (Bain \& Engelhardt, 1992), a CI can be constructed around the true value $\lambda$ :

$$
l_{i} \pm z^{*}\left(\sqrt{\frac{2 l_{i}^{2}}{n}}\right)
$$

Equation 9 has the benefit of being easy to calculate for any eigenvalue. The formula requires only three pieces of information that are always at hand: the appropriate $z$ value for the CI width $\left(z^{*}\right)$, each observed eigenvalue $\left(l_{i}\right)$, and the sample size $(n)$. Table 1 displays the CIs for the factors of the selected Holzinger and Swineford (1939) data that were calculated using Equation 9.

We have produced SAS and SPSS syntax for researchers who do not wish to calculate CIs by hand using Equation 9. The SAS syntax is part of our bootstrapping syntax supplied in Appendix A in the supplemental materials and is explained in detail in the next section of this article. Appendix B (also in the supplemental materials) contains the necessary SPSS syntax to calculate eigenvalue CIs using Equation 9. It is important to note that this SPSS syntax creates two small files, as is noted in the comments in the syntax. 


\section{Bootstrapping Method}

Bootstrapping is a statistical procedure used to empirically estimate a sampling distribution (Diaconis \& Efron, 1983). Bootstrapping requires the user to draw another sample of equal size $n$ as the original data by sampling with replacement. Resampling is repeated many times, and the researcher calculates statistics for each new sample. The distributions of each statistic are then compared with the original statistics of the entire sample to evaluate the probability of the statistics. Bootstrapping is an efficient procedure for examining replicability of statistics without collecting new data (Thompson, 2006).

Using this basic bootstrapping technique, it is possible to form a distribution of eigenvalues for each factor in a data set. These distributions are then used to create the appropriate CIs: The middle $95 \%$ of values, for example, constitute the $95 \% \mathrm{CI}$.

The accuracy of bootstrapping methods depends on a few assumptions. First, researchers assume that the sample is reasonably representative of the population. A biased sample will produce statistics that are also nonrepresentative of the population. In the case of bootstrapping eigenvalues, not only must the sample of participants be reasonably representative of the population, but the items or tests that are being factor analyzed must also resemble the theoretical universe that they represent. Researchers using bootstrapping also assume that their cases of data are independent. Violation of the independence assumption may produce biased results and create deceptively wide or narrow CIs.

In the next section, we will describe two syntax codes that we have adapted to produce CIs for eigenvalues. The two computer programs were SAS 9.2 and SPSS 17.0, which are recent releases of the two most popular statistical programs used by social scientists.

SAS. The SAS code that we present is adapted from Tonsor (2004) and is available at www.pitt.edu/ tonsor/ downloads/programs/PROGRAMS.html. Tonsor created the code in order to apply bootstrapping to many different aspects of factor analysis. Because we focus on CIs for eigenvalues in the present article, we have simplified the syntax in order to eliminate some of the other output that Tonsor's original code produces. We have also adapted the syntax to make it more user friendly for the social scientist.

Instructions for the user are found in the comments within the SAS code. Users must specify the number of observed variables, the original sample size, the percentage confidence limits wanted, and the number of iterations that the bootstrap must perform. The user also has the option of choosing between principal components analysis and principal axes analysis. The default that we have set is nine variables, 301 participants in the original sample, 1,000 resamplings, and principal components analysis.

The SAS code has the virtue of producing output that is easy to read. "PL1LCL" refers to a CI's lower limit for the first factor, whereas "PL1UCL" is the upper limit. The number before LCL or UCL refers to the factor number. Hence, "PL4UCL" would refer to the upper limit of the $\mathrm{CI}$ for the fourth eigenvalue in the data set. Between each lower limit and upper limit is the observed eigenvalue for the original sample, labeled "trait."

To change the width of the CI, the user must find the "percentile value" option for both the lower and upper limits of the CIs. We have set the default to a width of a $95 \%$ CI, but other CIs may be appropriate. Because there is an option for both the upper and lower limits, it is possible to have asymmetrical CIs, if the user so desires. To adjust the upper CI, the user should change the percentile rank values for the CIs (the defaults are 2.5 and 97.5 , producing a $95 \% \mathrm{CI}$ ). The correct place to do this in the SAS code is labeled with comments that read, "Set the lower confidence level for your interval," and "Set the upper confidence level for your interval."

SPSS. As was mentioned previously, the SPSS syntax we use was taken directly from Zientek and Thompson (2007). Like Tonsor's (2004) SAS syntax, Zientek and Thompson's SPSS syntax has other capabilities that we do not touch upon here, but that are explained fully in their article. To obtain a $95 \%$ CI, it is necessary to use the following syntax after performing the bootstrap procedure:

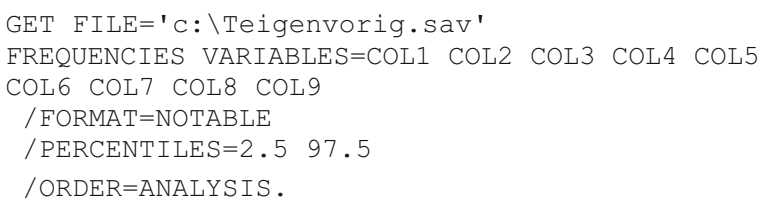

The variable names COL1 through COL9 correspond to the number of observed variables in the original data set. Users may need to eliminate or add variables to accommodate their data sets. The size of the CI is determined by the values after the percentiles subcommand. A $90 \%$ CI, for example, would correspond to the 5 th and 95th percentiles.

A user who prefers SPSS's point-and-click interface should open the Teigenvorig.sav file and select the "Frequencies" function from the "Analyze menu." In the dialogue box that appears, the user should click the "Statistics" button, which causes a second dialogue box to appear. In this box, the user should check the box marked "Percentiles" and manually enter the 2.5 th and 97.5 th percentiles for a $95 \%$ CI. Of course, whether one uses the point-and-click method or the syntax in the previous paragraph, nonsymmetrical CIs can be calculated by adjusting the percentile ranks accordingly.

Comparison between SAS and SPSS programs. Each syntax has its own advantages and disadvantages, and personal preference may determine whether a researcher uses the SPSS or SAS code. Our adaptation of Tonsor's (2004) code is much more computationally efficient than the SPSS syntax. On a 2.4-GHz Quad core PC, 1,000 resamplings using the SAS code took less than $2 \mathrm{~min}$. The same computer required $50 \mathrm{~min}$ to perform the same calculations using the SPSS code.

One issue that SPSS users should be aware of is that Zientek and Thompson's (2007) syntax creates 14 new SPSS data files in the course of its bootstrapping process, including the Teigenvorig.sav file needed to create the CIs. The SAS syntax, however, creates only two new files in the compiling process. This is because we have stripped 
Tonsor's (2004) code down to the bare essentials needed to calculate CIs for eigenvalues. The SPSS data files are not large (they are less than $1 \mathrm{MB}$ in total size in our example), but some users may not want to deal with them. Although it appears that using the SPSS code may not be advantageous for researchers, some may prefer to use it anyway because of their familiarity with the program. We have discussed both in the present article for the user's convenience and to make the use of bootstrapping to produce CIs possible for more readers.

\section{DISCUSSION}

Table 1 displays the CIs for the selected Holzinger and Swineford (1939) data that we have calculated with each method we have discussed in this article. As is apparent, the results are very similar for each of the three methods. Of course, the two bootstrap methods are susceptible to the idiosyncrasies of random resamplings. However, a greater number of resamplings in the bootstrap sequence should produce results that converge.

It should be noted that CIs produced through Equation 9 in our example tend to be wider than CIs produced through bootstrapping methods. It is unknown whether this is due to the characteristics of the original data, or whether the mathematical method we present always produces conservative CIs. The wider CIs may also be due to the data's being more dependent than is assumed by the formula. If this tendency for the bootstrapping method to produce narrower CIs is a consistent feature of the method, then bootstrapped CIs would have more statistical power when used in parallel analysis, the scree test, or the Guttman rule for determining the number of factors.

As was mentioned in the introductory section of the article, the use of CIs of eigenvalues in the Guttman rule, the scree test, and parallel analysis to determine the number of factors in a data set is relatively clear. This clarity is due to the fact that these methods of choosing the number of factors rely on observed eigenvalues. Other methods, such as Velicer's (1976) MAP test, or an examination of the magnitude of the residuals in the residual matrix (Thompson, 2004), do not use observed eigenvalues; rather, they rely on variations of the correlation matrix. This fundamentally different approach to choosing the number of factors means that eigenvalue CIs are less applicable to researchers using these methods of decision making.

However, we hope that the enhancements to traditional methods of determining the number of factors in an EFA will help researchers to be more thoughtful when choosing the number of factors they retain. We believe that decisions about the number of factors to retain will be more accurate when CIs are taken into consideration. Further research needs to be conducted to determine which method of calculating CIs is more accurate. Future researchers could also investigate the most appropriate way to apply eigenvalue CIs to a given factor retention decision, and whether any of the suggested uses of eigenvalue CIs outperform Velicer's (1976) MAP test and other methods that do not rely on observed eigenvalues.
We strongly advocate that researchers who use EFA report the CIs for their eigenvalues. We interpret current APA (2010) guidelines as requiring these CIs to be reported, and we hope that other researchers do so as well. In addition to being required by APA, we also believe that an awareness of CIs will lead to more accurate decisions about the number of factors to retain. Moreover, reporting CIs for eigenvalues increases the transparency that is essential to evaluating good research. We believe that reporting CIs for eigenvalues should be the rule, rather than the exception, in the 21 st century.

\section{AUTHOR NOTE}

The authors thank Bruce Thompson for his helpful feedback on earlier drafts of the manuscript and Stephen J. Tonsor, without whom this article would not be possible. Correspondence concerning this article should be directed to R. Larsen, Department of Educational Psychology, Texas A\&M University, TAMU 4225, College Station, TX 77843-4225 (e-mail: ross.larsen@dsmail.tamu.edu).

\section{REFERENCES}

American Psychological Association (2010). Publication manual of the American Psychological Association (6th ed.). Washington, DC: Author.

Bain, L., \& Engelhardt, M. (1992). Introduction to probability and mathematical statistics. Belmont, CA: Duxbury Press.

Cattell, R. B. (1966). The scree test for the number of factors. Multivariate Behavioral Research, 1, 245-276. doi:10.1207/ s15327906mbr0102_10

Costello, A. B., \& Osborne, J. W. (2005). Best practices in exploratory factor analysis: Four recommendations for getting the most from your analysis. Practical Assessment, Research \& Evaluation, 10, 1-9.

Cronbach, L. J. (1957). The two disciplines of scientific psychology. American Psychologist, 12, 671-684. doi:10.1037/h0043943

Cumming, G., \& Finch, S. (2005). Inference by eye: Confidence intervals and how to read pictures of data. American Psychologist, 60, 170-180. doi:10.1037/0003-066X.60.2.170

Diaconis, P., \& EFron, B. (1983). Computer-intensive methods in statistics. Scientific American, 248, 116-130.

Edgington, E. S. (1964). A tabulation of statistics used in psychology journals. American Psychologist, 19, 202-203. doi:10.1037/ h0039177

Edgington, E. S. (1974). A new tabulation of statistical procedures used in APA journals. American Psychologist, 29, 25-26. doi:10.1037/ h0035846

Elmore, P. B., \& Woehlke, P. L. (1988). Statistical methods employed in American Educational Research Journal, Educational Researcher, and Review of Educational Research from 1978 to 1987. Educational Researcher, 17, 19-20.

Fabrigar, L. R., Wegener, D. T., MacCallum, R. C., \& Strahan, E. J. (1999). Evaluating the use of exploratory factor analysis in psychological research. Psychological Methods, 4, 272-299. doi:10.1037/1082 $-989 X .4 .3 .272$

Ford, J. K., MacCallum, R. C., \& Tait, M. (1986). The application of exploratory factor analysis in applied psychology: A critical review and analysis. Personal Psychology, 39, 291-314. doi:10.1111/ j.1744-6570.1986.tb00583.x

GLORFELD, L. W. (1995). An improvement on Horn's parallel analysis methodology for selecting the correct number of factors to retain. Educational \& Psychological Measurement, 55, 377-393. doi:10.1177/0013164495055003002

Gorsuch, R. L. (1983). Factor analysis. Hillsdale, NJ: Erlbaum.

GorsucH, R. L. (2003). Factor analysis. In J. A. Schinka \& W. F. Velicer (Eds.), Handbook of psychology: Vol. 2. Research methods in psychology (pp. 143-164). Hoboken, NJ: Wiley.

GutTman, L. (1954). Some necessary conditions for common-factor analysis. Psychometrika, 19, 149-161. doi:10.1007/BF02289162

Hayton, J. C., Allen, D. G., \& Scarpello, V. (2004). Factor retention decisions in exploratory factor analysis: A tutorial on parallel 
analysis. Organizational Research Methods, 7, 191-205. doi:10.1177/ 1094428104263675

Henson, R. K., \& Roberts, J. K. (2006). Use of exploratory factor analysis in published research: Common errors and some comment on improved practice. Educational \& Psychological Measurement, 66, 393-416. doi:10.1177/0013164405282485

Holzinger, K. J., \& SwINEFORD, F. (1939). A study in factor analysis: The stability of a bi-factor solution. Chicago: University of Chicago Press.

Horn, J. L. (1965). A rationale and test for the number of factors in factor analysis. Psychometrika, 30, 179-185. doi:10.1007/BF02289447

Loehlin, J. C. (2004). Latent variable models (4th ed.). Mahwah, NJ: Erlbaum.

Mardia, K., Kent, J., \& BibBy, J. (1979). Multivariate analysis. New York: Academic Press.

O'CONNOR, B. P. (2000). SPSS and SAS programs for determining the number of components using parallel analysis and Velicer's MAP test. Behavior Research Methods, Instruments, \& Computers, 32, 396-402.

RENCHER, A., \& SCHIMEK, M. (1995). Methods of multivariate analysis. New York: Wiley.

RIPPE, D. D. (1953). Application of a large sampling criterion to some sampling problems in factor analysis. Psychometrika, 18, 191-205. doi:10.1007/BF02289056

Russell, D. W. (2002). In search of underlying dimensions: The use (and abuse) of factor analysis in Personality and Social Psychology Bulletin. Personality \& Social Psychology Bulletin, 28, 1629-1646. doi:10.1177/014616702237645

Spearman, C. (1904). "General intelligence," objectively determined and measured. American Journal of Psychology, 15, 201-293. doi: $10.2307 / 1412107$

THOMpson, B. (2004). Exploratory and confirmatory factor analysis: Understanding concepts and applications. Washington, DC: American Psychological Association.

Thompson, B. (2006). Foundations of behavioral statistics: An insightbased approach. New York: Guilford.
Tonsor, S. (2004). PCA bootstrap confidence intervals program [SAS code]. Retrieved from www.pitt.edu/ tonsor/downloads/programs/ bootpca.html.

Velicer, W. F. (1976). Determining the number of components from the matrix of partial correlations. Psychometrika, 41, 321-327. doi:10.1007/BF02293557

Wilkinson, L., \& The Task Force on Statistical Inference (1999). Statistical methods in psychology journals: Guidelines and explanations. American Psychologist, 54, 594-604. doi:10.1037/0003 -066 X.54.8.594

Zientek, L. R., Capraro, M. M., \& Capraro, R. M. (2008). Reporting practices in quantitative teacher education research: One look the evidence cited in the AERA panel report. Educational Researcher, 37 208-216. doi:10.3102/0013189X08319762

Zientek, L. R., \& Thompson, B. (2007). Applying the bootstrap to the multivariate case: Bootstrap component/factor analysis. Behavior Research Methods, 39, 318-325.

ZWICK, W. R., \& VELICER, W. F. (1982). Factors influencing four rules of determining the number of components to retain. Multivariate Behavioral Research, 17, 253-269. doi:10.1207/s15327906mbr1702_5

ZWICK, W. R., \& Velicer, W. F. (1986). Comparison of five rules for determining the number of components to retain. Psychological Bulletin, 99, 432-442. doi:10.1037/0033-2909.99.3.432

\section{SUPPLEMENTAL MATERIALS}

The data from Holzinger and Swineford (1939) used for the analysis reported in Table 1, as well as SAS and SPSS syntax to calculate confidence intervals for eigenvalues, may be downloaded from http://brm .psychonomic-journals.org/content/supplemental.

(Manuscript received October 16, 2009 revision accepted for publication April 5, 2010.) 